\title{
MODELING FAULTS OF QUADCOPTER AERIAL VEHICLE USING PETRI NETS
}

\author{
Ailet Abreu López ${ }^{1}$, Julio Cesar Bravo Cortés ${ }^{2}$, José Rafael Abreu García ${ }^{3}$, Eduardo Izaguirre \\ Castellanos $^{4}$
}
1,3,4 Departamento de Control Automático, Facultad de Ingeniería Eléctrica, Universidad Central “Marta Abreu” de Las Villas, Santa Clara, Villa Clara.
${ }^{2}$ UEB Hidroeléctrica Hanabanilla dirección: Salto de Hanabanilla, Manicaragua, Villa Clara.

Email: aileta@uclv.cu, julio@hab.hidroe.une.cu,abreu@uclv.edu.cu,izaguirre@uclv.edu.cu

Received: April 04 ${ }^{\text {th }}, 2019$

Accepted: April 25th 2019

Published: June $30^{\text {th }}, 2019$

Copyright (02016 by authors and Institute of Technology Galileo of Amazon (ITEGAM). This work is licensed under the Creative Commons Attribution International License (CC BY 4.0).

https://creativecommons.org/lice nses/by/4.0/

\begin{abstract}
The development of models focused on the detection and diagnosis of failures in autonomous aerial vehicles, constitutes a novel subject, where for this application context the complexity of the algorithms and sensorial systems on board of these vehicles constitutes a challenge. In this field Petri Networks are used as a tool for fault detection given the facilities they provide for the modeling of complex systems in terms of their graphic representation and offering a simple solution. In this investigation a fault model is developed that allows the detection of faults based on Petri Nets applied to an air vehicle type Quadcopter, this model allows to determine the possible existence of off-line faults for this type of aerial vehicle, through the analysis of the data coming from the flight controller and measurements of the sensors on board. It is shown that the model satisfies the properties established for the verification of models with Petri Nets using two of the methods; reachability tree and state equation.
\end{abstract}

Keywords: Modeling, Petri Nets, Aerial Vehicles, Fault Detection.

\section{MODELADO DE FALLOS DE VEHÍCULO AÉREO QUADCOPTER UTILIZANDO REDES DE PETRI}

\section{RESUMEN}

El desarrollo de modelos enfocados a la detección y diagnóstico de fallos en vehículos autónomos aéreos, constituye un tema novedoso, donde para este contexto de aplicación la complejidad de los algoritmos y sistemas sensoriales a bordo de estos vehículos constituye un desafío. En este campo las Redes de Petri se emplean como herramienta para la detección de fallos dados las facilidades que estas brindan para el modelado de sistemas complejos en cuanto a su representación gráfica y que ofrecen una solución sencilla. En esta investigación se desarrolla un modelo de fallos que permite la detección de fallos basado en Redes de Petri aplicado a un vehículo aéreo tipo Quadcopter, dicho modelo permite determinar la posible existencia de fallos fuera de línea para este tipo de vehículo aéreo, mediante el análisis de los datos provenientes del controlador de vuelo y mediciones de los sensores a bordo. Se demuestra que el modelo satisface las propiedades establecidas para la verificación de modelos con Redes de Petri haciendo uso de dos de los métodos; árbol de alcanzabilidad y ecuación de estado.

Keywords: Modelado, Redes de Petri, Vehículos Aéreos, Detección de Fallos. 


\section{INTRODUCCIÓN}

Un vehículo aéreo no tripulado (VANT), es una aeronave que vuela sin tripulación. Los VANT se usan tanto en el ámbito civil como militar y pueden tener diferentes formas, tamaños, configuraciones y características. Se pueden controlar de forma remota y poseen la capacidad de desarrollar misiones de forma autónoma, siendo capaces de despegar, volar y aterrizar sin la intervención humana. [1]

La utilización de los VANT tiene un gran número de ventajas respecto de los vehículos aéreos tripulados.[1], entre ellas su uso en zonas de alto riesgo o difícil acceso, y la no existencia de un piloto a bordo de la aeronave, por lo que no hay riesgo para su vida facilitando la realización de movimientos con aceleraciones. Entre las desventajas, la aparición de fallos que pueden afectar el desempeño de la misión [2]-[4], la limitación en el tiempo de vuelo por razones energéticas [5] y posibilidad de ser hackeados o neutralizados, siendo vulnerables ante afectaciones de los canales de comunicación [6].

Dada sus complejidades, su estudio resulta un gran reto, donde la posibilidad de la detección de fallos constituye un elemento fundamental a la hora de evitar roturas y averías que pudieran poner en peligro la misión asignada al vehículo. Por tanto, se hace necesario definir los conceptos de fallos y averías:

Fallo: es una desviación de al menos una característica o propiedad (comportamiento) del sistema de una condición estándar o de un comportamiento deseado [3].

Avería: es una interrupción permanente que impide al sistema mantener la capacidad de comportarse bajo ciertas especificaciones de operación.

Varios autores han investigado en el campo de la detección de fallos en VANTs. En la investigación reportada por Bauer [7], se presenta un sistema de detección de fallos mecánicos en actuadores aplicado en vuelo a un vehículo aéreo no tripulado, empleándose una estimación adaptativa de modelos múltiples, ello exige el modelo matemático del vehículo, lo que resulta engorroso dadas las complejidades de estos sistemas.

Zhong y colaboradores [8] presentan un esquema robusto de detección y diagnóstico de fallos para un Quadcopter, construyen el modelo dinámico teniendo en cuenta las perturbaciones externas y los fallos en actuadores, desarrollando un estado aumentado adaptativo de Kalman (AASKF). A pesar de que este método brinda buenos resultados, exige una elevada carga computacional, y la realización del modelo dinámico y de los filtros de Kalman implican un análisis matemático profundo.

Otros investigadores reportan estudios dirigidos a fallos en sensores y actuadores, tal es el caso del algoritmo basado en aprendizaje profundo propuesto por Olyaei [9] para detectar e identificar fallos en un VANT utilizando imágenes obtenidas de gráficos de amplitud tiempo y frecuencia, donde el procesamiento y clasificación de los fallos se basa en imágenes que utilizan redes neuronales.

Existen trabajos donde las cámaras visuales se emplean para la detección e identificación de fallos en grupos de VANT, las mismas se usan como sensores para estimar la orientación relativa de otro VANT. En este caso la detección de fallos se basa en la generación de residuos que comparan la posición esperada de un vehículo, considerado como objetivo, con las mediciones tomadas por uno o más VANTs [10]. Ello implica exigencias computacionales para el procesamiento de la información y la necesidad de emplear múltiples vehículos con cámaras.

En estas investigaciones generalmente se requiere del modelo modelado dinámico del vehículo, por demás complejo dadas las características de estos sistemas, principalmente por la dinámica del vehículo y además por los sensores que se integran a él como GPS, giróscopo, acelerómetro, magnetómetro, así como el hardware compuesto por los motores, controladores electrónicos de velocidad, controlador de vuelos, entre otros.

Es por ello que varios investigadores se auxilian de las Redes de Petri (RdP) como herramienta matemática para el modelado de sistemas, dadas las ventajas en cuanto a la representación gráfica de las $\mathrm{RdP}$, que facilita el trabajo con sistemas caracterizados por modelos dinámicos complejos. En este campo se reportan aplicaciones en el modelo de sistemas físicos [11], sistemas de potencia [12]-[14], sistemas de eventos discretos [15]-[18], síntesis e identificación de modelos de fallos [19], etc.

Desde el punto de vista de la aplicación de las RdP para el modelo de fallos en VANT, se reporta la contribución de Bertolaso y colaboradores, que realizan [20] un modelo con RdP aplicado a una misión asignada a un Quadcopter y a un vehículo terrestre no tripulado, utilizando Redes de Petri Planificadas. En otra aplicación, se destaca la utilización de las RdP en la construcción de un modelo que abarca los principales subsistemas que componen a un helicóptero no tripulado, en condiciones de vuelo normal y en fallo, planteándose una metodología para el modelado de fallos con el uso de las RdP, que brinda con mayor claridad en la concepción del complejo modelado dinámico [21].

Teniendo en cuenta la importancia que reviste la detección de fallos en estos vehículos y las facilidades que brindan las Redes de Petri (RdP) como herramienta para la construcción del modelo de fallos, atendiendo a sus ventajas en el modelado de sistemas complejos y que resulta un tema novedoso aplicado a la detección de fallos en Quadcopter, se propone, como objetivo de esta investigación, desarrollar el modelo de fallos basado en RdP aplicado al Quadcopter X4 GARP, con el propósito de realizar un análisis de los fallos que ocurren al vehículo. Para el modelado se sigue la metodología propuesta por [22] siendo esta una metodología probada para estos vehículos. En esta se divide el sistema en subsistemas permitiendo evaluar los fallos de manera independiente. Además, la metodología propuesta por el autor brinda la capacidad de ser flexible a cambios pudiéndose incluir otros fallos que se reporten en el futuro.

El documento está estructurado de la siguiente forma, en el primer epígrafe se realiza una descripción del vehículo teniendo en cuenta los principales subsistemas que lo componen y los fallos generales asociados a estos, posteriormente se presenta la metodología a seguir para la construcción del modelo, en el tercer epígrafe se inicia el modelado con RdP de cada uno de los componentes del sistema, teniendo en cuenta el comportamiento normal y de fallo del vehículo, finalmente a este modelo se le aplican dos métodos de validación de modelos.

\section{DESCRIPCIÓN DEL QUADCOPTER X4 GARP}

Los VANT pueden clasificarse de varias formas, entre ellas por sus alas encontrándose los vehículos de ala fija y los de ala rotatorias, estos últimos están compuestos generalmente por cuatro motores con sus hélices pudiendo llegar hasta ocho o más motores. Destacan por sus ventajas entre ellas el despegue y aterrizaje en vertical, posibilidad de volar en un punto fijo y mayor precisión y maniobrabilidad. [23]. El Quadcopter objeto de aplicación en esta investigación es el X4 GARP perteneciente al laboratorio de investigaciones del Grupo de Automática, Robótica y Percepción de la Universidad Central "Marta Abreu" de Las Villas. 


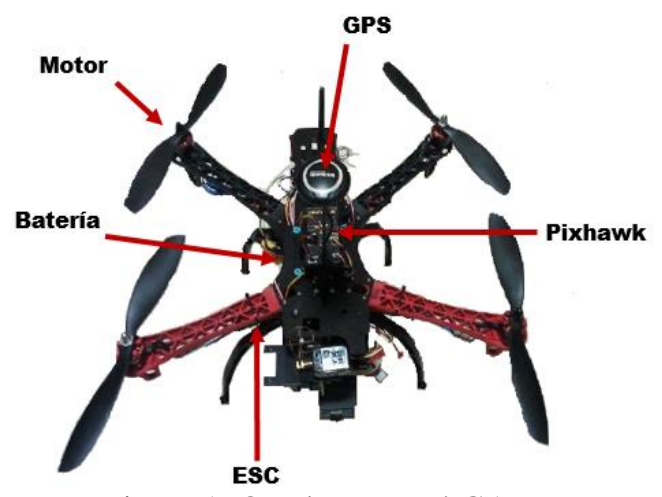

Figura 1: Quadcopter X4 GARP.

Tabla 1: Componentes fundamentales del Quadcopter X4 GARP.

\begin{tabular}{|c|c|}
\hline Motores & Modelo DJI 2212-920 Kv \\
\hline $\begin{array}{c}\text { Controlador } \\
\text { Electrónico de } \\
\text { Velocidad }\end{array}$ & Modelo Simonk \\
\hline $\begin{array}{c}\text { Controlador de } \\
\text { Vuelo }\end{array}$ & $\begin{array}{c}\text { 3DR PX4 Pixhawk, incorpora } \\
\text { una unidad inercial de medición } \\
\text { IMU }\end{array}$ \\
\hline Batería & 3DR 4S 6000 mAh Voltaje \\
& Nominal 16.8 V \\
\hline
\end{tabular}

A lo largo de las experiencias acumuladas de las diferentes misiones de vuelo, el vehículo ha visto en varias ocasiones comprometida su misión producto de que se han presentado varios fallos que han traído como consecuencia la interrupción de misiones importantes y un arduo trabajo para determinar el motivo que provoco el fallo. Es por ello, que resulta de gran importancia buscar una vía que permita la determinación de la posible existencia de fallos fuera de línea para este tipo de vehículo aéreo, con vistas a tomar decisiones en relación con el cumplimiento de la misión de vuelo asignada.

\section{DESARROLLO DEL MODELO}

Previo a la aplicación de la metodología para la construcción del modelo, se deben tener en cuenta las siguientes consideraciones:

- El Quadcopter debe ser encendido de forma manual.

- Se asume que ningún fallo ocurre en el algoritmo que se ejecuta en el Controlador de Vuelo, es decir no ocurren errores por concepto de programación.

- No existen fallos en el Sistema de Comunicación con la estación en tierra.

\section{Paso 1. Dividir el Sistema en Subsistemas}

El sistema denotado por $H$ puede ser dividido en $M$ subsistemas con relación entre ellos[21], [22], dependiendo de su comportamiento. Esto permite reducir la complejidad de los sistemas. Así:

$$
H_{j}=H_{1} \cup H_{2} \cup \ldots \cup H_{k} \quad \forall j=1 \ldots k
$$

En este sentido, se propone dividir el VANT en cinco subsistemas principales, estos son: Motor, Controlador Electrónico de Velocidad (ESC), Batería, módulo GPS e IMU, los cuales se describen a continuación.

Batería $\left(\mathrm{H}_{1}\right)$ : es el encargado de suministrar la potencia a los motores, a los sensores y controlador de vuelo del Quadcopter. Está compuesto por una batería LiPo de 16.8 V y 6000 mAh.
Uno de los principales problemas de los VANT lo constituye el sistema de alimentación de energía. Dada la construcción de los motores y la presencia de bobinas en los mismos, la fuente de alimentación debe suministrar unas intensidades de corrientes relativamente grandes para lo que suelen dar las baterías estándar.[4].

Un descenso en el voltaje de la batería durante la operación del quadcopter podría conducir al mal funcionamiento del hardware. Las baterías LiPo tienen ciertos inconvenientes. Estas aparte de funcionar mal a temperaturas altas o bajas, tienen un voltaje mínimo, de unos $3 \mathrm{~V}$ por celda [12]. El quadcopter posee un módulo de alimentación que se conecta a la batería y alimenta al Pixhawk con un voltaje de $5.3 \mathrm{~V}$. Las variaciones del voltaje de alimentación pueden provocar una disminución del suministro de voltaje al Pixhawk.

Controlador Electrónico de Velocidad (ESC) $\left(\mathrm{H}_{2}\right)$ : son cuatro, uno por cada motor del Quadcopter, son los encargados de convertir la corriente DC suministrada por la batería en una corriente trifásica que mueve los motores sin escobilla. Los controladores electrónicos de velocidad que utiliza el VANT son el modelo Simonk con una corriente máxima de 30 A, ideal para baterías de 2 a 4 celdas de LiPo.

Estos dispositivos son, junto con los motores, unos de los más complejos debido a la importancia que reviste su correcto funcionamiento, de ellos depende el trabajo que realicen los motores y como ha ocurrido en ocasiones un fallo en uno de los ESC ha provocado un desbalance en el vehículo llevando al descontrol del mismo y por ende la caída del vehículo y la perdida de la misión. Si el motor requiere más corriente de la que el ESC puede proporcionarle, este se calentará y acabará fallando.

Motor $\left(\mathrm{H}_{3}\right)$ : son cuatro motores sin escobilla accionados por sus respectivos ESC. Los motores son los encargados de darle suspensión y movimiento en cualquier dirección según la variación de voltaje, a la aeronave. Estos motores están diseñados para desarrollar una potencia máxima de $370 \mathrm{~W}$, valor de Kv de 920 $\mathrm{rpm} / \mathrm{V}$, consumen una corriente estándar entre 15-25 A y corriente máxima de $30 \mathrm{~A}$. La temperatura de trabajo está entre $-5^{\circ} \mathrm{C}$ y $40^{\circ} \mathrm{C}$.

Los componentes de este subsistema se caracterizan por ser uno de los más propensos a fallos: los mecánicos como es el caso de los fallos por desgaste operacional, conducen a una generación de empuje insuficiente y traen consigo inestabilidad en el vehículo o la pérdida del control; los fallos por calentamiento pueden provocar el mal funcionamiento o destrucción de los motores, y puede ocurrir si la temperatura del motor sobrepasa el valor máximo correspondiente a $40{ }^{\circ} \mathrm{C}$. Aunque el incremento de la temperatura no afecte el correcto funcionamiento del motor durante un tiempo, puede dañar componentes del motor [3] y provocar un posible fallo.

Unidad Inercial de Medición (IMU) $\left(\mathrm{H}_{4}\right)$ : integrada en el controlador de vuelo 3DR PX4 Pixhawk, considerándose un subsistema aparte por su función. Los sensores integrados en su circuito (giróscopo (G) 3-ejes, acelerómetro (A) 3-ejes y un magnetómetro (M)) son encargados de medir posición, velocidad y aceleración del vehículo.

Este subsistema puede verse afectado por la velocidad de giro de los rotores y las vibraciones generadas en la estructura, que en principio pueden parecer despreciables, tienen un gran efecto en las medidas que recogen los sensores, distorsionándolas hasta el punto de dificultar el control del Quadcopter. En específico, las mediciones del acelerómetro y el giróscopo son propensas a una desviación (bias) debido a estas afectaciones. Las mediciones de la brújula se afectan por campos magnéticos externos.

Módulo GPS ( $\left.\mathrm{H}_{5}\right)$ : está compuesto por el GPS y una brújula digital integrada Ublox M8N. Este subsistema es el encargado de posicionar la aeronave y de calcular la altura a la que se encuentra, 
a partir de la señal recibida de distintos satélites. Se obtiene un bloqueo cuando el GPS recibe datos de posicionamiento de múltiples satélites. La conexión a cuatro satélites activos produce un bloqueo del GPS.

Para volar con precisión, se requiere una señal de GPS fuerte. Hay dos mediciones importantes de la señal del GPS: el bloqueo del GPS (magnitud de la señal) y la incertidumbre en la medida horizontal (HDOP) que brinda una medida de la precisión de la señal GPS. La fuerza y la precisión de la señal GPS pueden verse afectadas por la falta de satélites disponibles y/o por las posiciones de los satélites, además de obstrucciones como edificios y montañas, efectos atmosféricos e interferencia electromagnética de torres de radiofrecuencia u otras.

\section{Paso 2. Elaboración del Modelo de RdP de los componentes de cada subsistema}

En los modelos se tiene en cuenta el comportamiento normal y de fallo. Al mismo tiempo se tienen que definir los fallos de los componentes en cada subsistema.

Sea $R_{j}^{i}=\left(P, T, P_{r e}, P_{o s t}, M_{0}\right)$ la RdP marcada del $i$ componente correspondiente al subsistema $j$. Por consiguiente a cada subsistema $\mathrm{H}_{\mathrm{j}}$, le corresponde una red de Petri $R_{j}^{i}$. Donde los vectores $\mathrm{P}$ y $\mathrm{T}$ representan la cantidad de estados y transiciones de la RdP de cada j-subsistema, donde se define:

n: número de estados del j-subsistema

$$
\mathrm{P}=\left[\mathrm{P}_{1}, \mathrm{P}_{2}, \ldots \mathrm{P}_{\mathrm{n}}\right]
$$

$$
\mathrm{T}=\left[\mathrm{T}_{1}, \mathrm{~T}_{2}, \ldots \mathrm{T}_{\mathrm{m}}\right]
$$

$\mathrm{m}$ : número de transiciones del $\mathrm{j}$-subsistema

$P_{\text {re }} / P_{\text {ost }}$ son las aplicaciones de la incidencia de entrada/salida y $M_{0}$ es el marcado inicial [21].

A continuación, se procede a la elaboración del modelo de RdP de los elementos definidos de cada subsistema.

$\boldsymbol{H}_{\mathbf{1}}$ : El subsistema tiene como componente a modelar la batería LiPo $H_{1}^{1}$, la misma brinda un voltaje máximo de $16.8 \mathrm{~V}$, el cual puede comprometer la seguridad del vuelo cuando su magnitud cae por debajo de $12 \mathrm{~V}$ (condición de fallo). Por consiguiente, la variable a evaluar se define como Vbat (voltaje de la batería).

Para el sistema bajo estudio, según las prácticas de vuelo realizadas y criterios de especialistas se fija un valor de $12.3 \mathrm{~V}$ como el valor mínimo suficiente para que el quadcopter pueda retornar al punto de lanzamiento. Bajo esta condición el fallo a modelar es Fallo en el Suministro de Energía (FSE), quedando establecido el modelo de RdP de este subsistema en el esquema de figura 2-a.

$\boldsymbol{H}_{2}$ : El subsistema representa a los ESC $H_{2}^{1}$ como componentes a modelar. El mismo suministra la potencia necesaria para alimentar los motores de la aeronave, donde el rango permisible de voltaje de salida debe estar entre 7 y $12 \mathrm{~V}$. En este subsistema la variable a evaluar se establece como Vout (voltaje de salida del ESC).

En este sentido, el fallo a modelar se denomina Fallo en el Nivel de Voltaje (FNV) que aparecerá en caso de no cumplirse el límite de voltaje mínimo especificado (Vout $<7$ volts DC). En la figura 2-b se muestra como queda conformado el modelo de RdP de este subsistema.
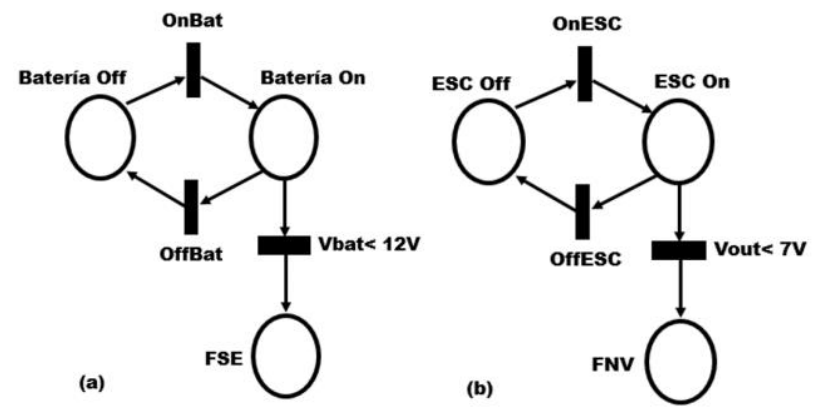

Figura 2: Modelos de RdP para los subsistemas Batería (a) y ESC (b) representando el comportamiento normal y de fallo.

$\boldsymbol{H}_{3}$ : El componente a modelar es el motor (son cuatro, pero como son idénticos se modelará uno). Trabajan en un rango de temperatura de -5 a $40{ }^{\circ} \mathrm{C}$ y con velocidades entre $11510 \mathrm{rpm}$ (12 V) a $6720 \mathrm{rpm}(7 \mathrm{~V})$. Por lo que las variables que interviene se definen como Tm (Temperatura de trabajo del Motor) si Tm $>40^{\circ} \mathrm{C}$ sería considerado como una condición de fallo y Veloc (Velocidad del Motor) requiriendo a su salida una velocidad mínima de 6720 rpm y una velocidad máxima de $11520 \mathrm{rpm}$.

En consecuencia, los fallos a modelar son Fallo por Calentamiento del Motor (FCM) que aparece al sobrepasar los $40^{\circ} \mathrm{C}$ de temperatura y Fallo por Atascamiento del Motor (FAM) que depende de la velocidad mínima de $6720 \mathrm{rpm}$ que se corresponde con el valor de voltaje mínimo con que trabaja el motor. En caso de disminuir la velocidad de los motores por debajo de este valor umbral aparece un fallo.

$\boldsymbol{H}_{\mathbf{4}}$ : El subsistema está representado por la IMU. Esta tiene integrado los tres sensores básicos de una IMU. El elemento a validar será el funcionamiento correcto de cada uno de los sensores que la conforman. Por consiguiente, las variables se definen como A (Acelerómetro), G (Giróscopo), M (Magnetómetro).

De acuerdo con lo anterior el fallo a modelar se define Fallo en los Sensores de la IMU (FS). De no validarse las lecturas en cada sensor se presenta una condición de fallo.

$\boldsymbol{H}_{\mathbf{5}}$ : está compuesto por el módulo GPS, este funciona según la conexión que posea con varios satélites, el elemento a validar es si existe o no conexión del módulo con los cuatro satélites, por tanto, la variable denominada es Cant. Satélites (Cantidad de Satélites).

Por consiguiente, el fallo a modelar es Fallo en la Medición de Altura (FMA) la conexión del módulo GPS con cuatro satélites es necesaria, si no existe esta conexión aparece una condición de fallo.

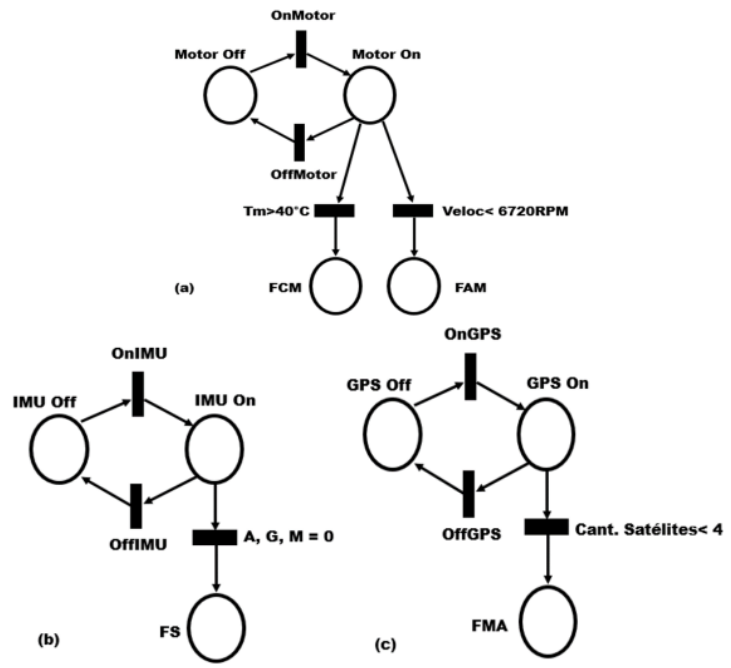

Figura 3: Modelo RdP de los subsistemas Motor (a), IMU (b), Módulo GPS (c). 


\section{Paso 3. Operación de integración}

Para denotar la operación de integración de los subsistemas se emplea la expresión $\widetilde{Q^{J}}=\left(\widetilde{P^{J}}, \widetilde{T^{J}}, \widetilde{P_{r e}{ }^{j}}, \widetilde{P_{o s t}{ }^{J}}, \widetilde{M_{0}{ }^{j}}\right)$, donde $\widetilde{Q^{J}}$ representa el comportamiento del subsistema $H_{j}$ a través de un único modelo de RdP, el cual incluye $N_{j}$ diferentes modelos de la red correspondientes a sus componentes. El mismo integra el comportamiento normal y de fallo del sistema, las transiciones (observables $T_{o}$ y no observables $T_{o u}$ ) que pueden ocurrir en cualquier lugar.

Para el VANT objeto de esta investigación no es necesario integrar el modelo de cada subsistema debido a que los lugares normales están unidos y los de fallos están bien identificados. Por lo que se procede con la refinación del modelo general, que se describe en el siguiente paso.

\section{Paso 4. Refinación del Modelo General}

Es necesario considerar solo la parte observable del modelo general $\widetilde{Q^{J}}$. Por lo tanto, $\widetilde{Q^{J}}=\left(\widetilde{P^{\jmath}}, \widetilde{T^{\jmath}}, \widetilde{P_{r e}{ }^{J}}, \widetilde{P_{o s t}{ }^{J}}, \widetilde{m_{0}{ }^{J}}\right)$ debe ser transformado al modelo general refinado $Q^{j}=$ $\left(P^{j}, T^{j}, P_{r e}^{j}, P_{o s t}^{j}, m_{0}^{j}\right)$. Este modelo está solo compuesto por lugares y transiciones observables. Acorde a esto, las transiciones de fallo tienen que ser reemplazadas por lecturas de los sensores, luego para refinar el modelo se procede con los siguientes pasos:

\section{Paso 4.1 Identificar los Sensores $N_{S}$ para cada subsistema}

Esto se hace acorde a los sensores requeridos para la monitorización de las variables deseadas. El conjunto de sensores del Motor está compuesto por el sensor de temperatura (T) y el sensor de velocidad (RPM); por tanto el conjunto $N_{S}$ para el subsistema motor está definido como: $N_{S 1}=T, R P M$. Siguiendo el mismo procedimiento el conjunto de sensores del ESC está compuesto solo por el sensor de voltaje $\left(V_{\text {out }}\right)$, siendo $N_{S 2}=$ $V_{\text {out }}$. Mientras el conjunto de sensores de la IMU está dado por: Giróscopo $(G)$, Acelerómetro $(A)$ y Magnetómetro $(M)$; por tanto $N_{s 3}=G, A, M$. El conjunto de sensores del subsistema Batería está compuesto solo por sensor de voltaje de la batería $\left(V_{b a t}\right)$, siendo $N_{s 3}=V_{b a t}$. Por último el subsistema Módulo GPS está compuesto, para el caso particular de esta investigación, por el GPS; $N_{s 3}=G P S$.

Paso 4.2 Construir el Conjunto Discreto de Salidas de los Sensores Cada sensor del sistema entregará una medida discreta que define su estado; con estas medidas se construye el conjunto $Y$ de salidas de los sensores y permite establecer con cuales lecturas se cuenta en la evaluación de cada subsistema.

Subsistema Motor: compuesto por el sensor de velocidad (RPM) y el sensor de temperatura (T), $|Y|=2^{N s}=2^{2}=4$ combinaciones.

Subsistema ESC: compuesto por el sensor de voltaje salida del $\operatorname{ESC}\left(V_{\text {out }}\right)$, siendo $|Y|=2^{1}=2$.

Subsistema IMU: compuesto por acelerómetro, giróscopo y magnetómetro, teniendo $|Y|=2^{3}=8$.

Subsistema Batería: compuesto por el sensor de voltaje batería $\left(V_{\text {bat }}\right)$, siendo $|Y|=2^{1}=2$.

Subsistema Módulo GPS: compuesto por el GPS, teniendo $|Y|=2^{1}=2$.

Paso 4.3 Definir las Salidas de la Tabla de Integración de Sensores

Para cada lugar del Modelo General RdP de cada subsistema se agrega una salida en la tabla de integración de sensores, esta salida contiene la representación de las lecturas del subsistema en un estado de funcionamiento normal (N).

Subsistema Motor: dos salidas han sido adicionadas para el subsistema $\left(P_{3} y P_{4}\right)$. Bajo condiciones de funcionamiento normal (N) el estado de la lectura de temperatura $\bar{T}$ (temperatura del motor está por debajo de $\operatorname{los} 40^{\circ} \mathrm{C}$ ) y el estado de velocidad del motor debe ser las RPM (las revoluciones por minuto están por encima del umbral mínimo 6720 RPM).

Subsistema IMU: dos salidas han sido definidas para este subsistema $\left(P_{9}\right.$ y $\left.P_{10}\right)$. Bajo condiciones de operación normal la lectura del acelerómetro será $A$ (correcta lectura aceleración en 3 ejes), la lectura del giróscopo $G$ (correcta lectura de la velocidad angular en 3 ejes) y la lectura del Magnetómetro $M$ (correcta lectura de orientación magnética).

Subsistema ESC: dos salidas han sido definidas para este subsistema $\left(P_{5} y P_{6}\right)$. Bajo condiciones normales de funcionamiento la lectura de voltaje de salida del ESC $V_{\text {out }}$ (voltaje de salida del ESC por encima de los 7V).

Subsistema Batería: dos salidas han sido definidas para este subsistema $\left(P_{7} y P_{8}\right)$ Bajo condiciones normales de funcionamiento la lectura de voltaje de batería $V_{b a t}$ (voltaje de la batería por encima de los $12 \mathrm{~V}$ ).

Subsistema GPS: dos salidas han sido incorporadas a la tabla $\left(P_{11} y P_{12}\right)$. La lectura del GPS deber estar en el GPS (señal de 4 satélites adquirida por el módulo GPS) para que esté en funcionamiento correcto.

\section{Paso 4.4 Construcción de las tablas de integración de sensores}

Las lecturas de los sensores $(Y)$ tienen que verificarse de manera cruzada con la correspondiente lectura de funcionamiento normal de cada subsistema. Las lecturas de los sensores permiten identificar qué lectura de los sensores es Normal $(N)$, de Fallo $(F)$ o es una lectura que no brinda información útil alguna $(X)$. Este paso requiere un profundo conocimiento del sistema.

En el subsistema Motor se utiliza la información de cada sensor, solo o en unión con los otros sensores para determinar los posibles fallos, por ello, estando el subsistema Motor en el estado $P_{4}$ sus lecturas de comportamiento normal son $(\bar{T}, \mathrm{RPM})$. Si en algún momento estas lecturas cambian mostrando por ejemplo; que exista o no calentamiento del motor $T$, esto significa que ha ocurrido un Fallo por Calentamiento del Motor, por el contrario, cuando las lecturas de los sensores coinciden con las esperadas, se registra en la tabla como un evento normal. Del mismo modo se realizan todas las combinaciones y lecturas de los demás sensores.

\section{Paso 4.5 Remplazar las Transiciones de Fallo y Eliminar los Lugares de Fallo No Alcanzables}

Este paso consiste en remplazar las transiciones no observables (transiciones de fallo) por lecturas sensoriales identificadas en la tabla de integración de sensores.

Subsistema Motor: en el modelo de RdP se procede a sustituir la transición de FCM por la lectura de temperatura $(T)$ y la transición de FAM por la lectura de velocidad ( $R P M)$.

Subsistema IMU: el subsistema IMU depende del funcionamiento de los tres sensores integrados: Acelerómetro, Giróscopo y Magnetómetro, por ello en el modelo de RdP se sustituyen la transición FS por el producto de las lecturas de los sensores $A, G, M$.

Subsistema ESC: como el funcionamiento del ESC depende de la lectura de voltaje a la salida del mismo, se procede a sustituir la transición FNV por la lectura del sensor $V_{\text {out }}$. 
Subsistema Batería: la lectura de voltaje de la batería es la que identifica el funcionamiento del subsistema, entonces se sustituye la transición FSE por la lectura del sensor de voltaje de la batería $V_{\text {bat }}$.

Subsistema Módulo GPS: en el modelo de RdP se ha sustituido el FMA por la lectura del GPS.

Redes de Petri Refinadas para cada subsistema:
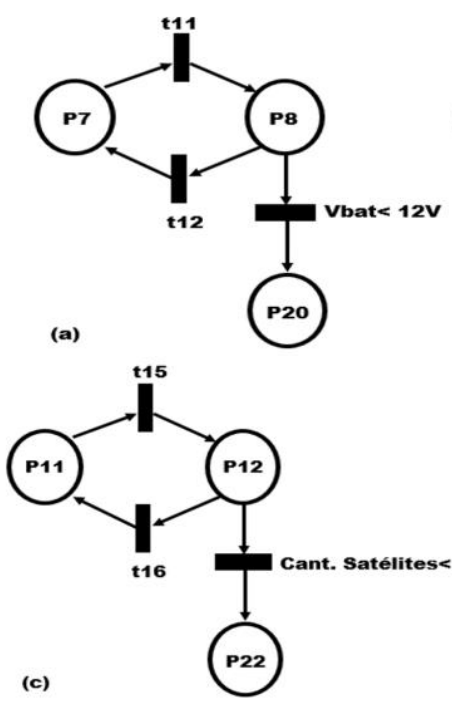

(b)
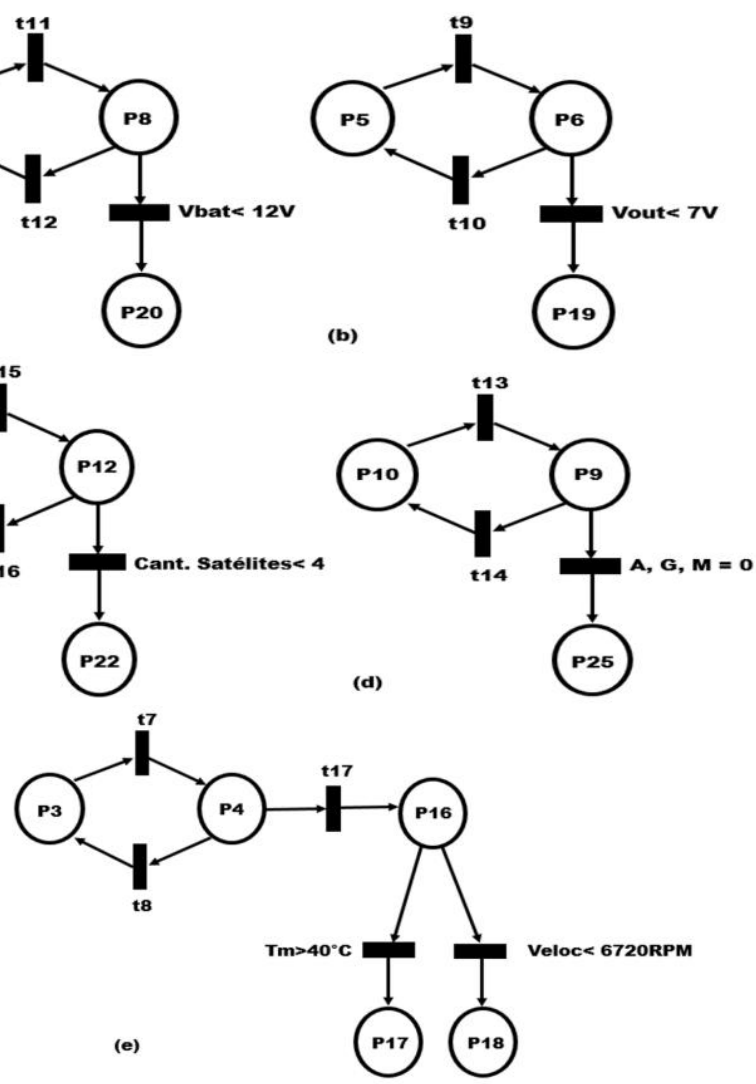

Figura 4: Modelos de RdP refinados para los subsistemas Batería (a), ESC (b), Módulo GPS (c), IMU (d) Motor (e).

Finalmente, se integra el modelo quedando como se muestra en la figura 5. En este modelo integrado se realiza un análisis antes del vuelo y después de estar el vehículo volando, además se incluyen las relaciones existentes entre los subsistemas al influir un fallo en otro subsistema. Con este se podrá implementar futuramente un diagnosticador de fallos que permita realizar el análisis de vuelos con el fin de determinar los fallos acontecidos, el lugar en el que ocurrió el fallo y las posibles causas.

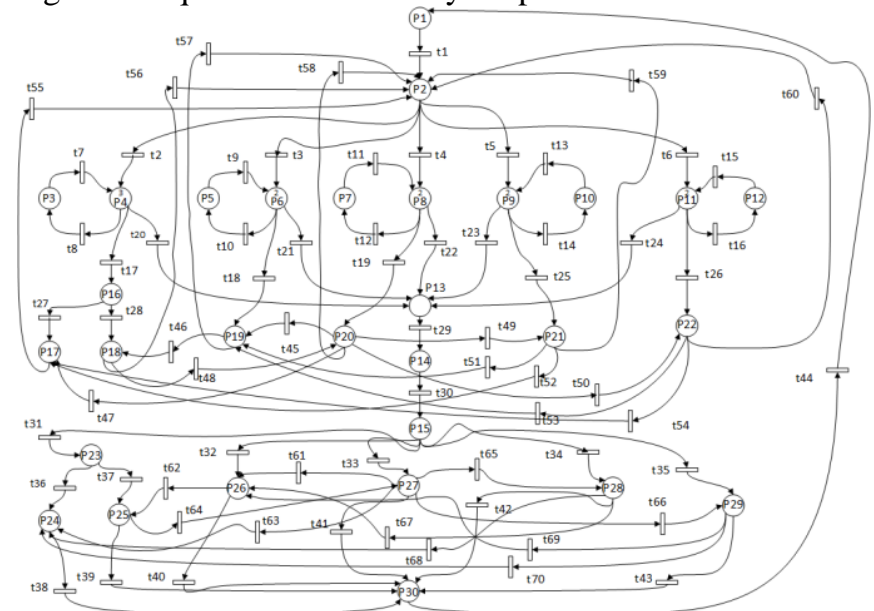

Figura 5: Modelo Integrado de RdP compuesto por los modelos de los subsistemas y las relaciones existentes entre los fallos.

\section{RESULTADOS}

Finalmente, para corroborar el modelo con RdP se procede a validar el modelo mediante la comprobación de ciertas propiedades que deben ser satisfechas. [24]

Los principales métodos de análisis para la verificación son:

- Análisis por reducción o transformación.

- Árbol de alcanzabilidad.

- Método de Eliminación de Gauss-Jordan.

- Ecuación de Estado.

- Método del Teorema del rango para redes de libre elección.

A continuación, se emplearán las técnicas de Árbol de Alcanzabilidad y Ecuación de Estados para verificar el cumplimiento de algunas propiedades de la red.

Árbol de Alcanzabilidad

Este método toma como raíz el marcado inicial de la RdP y, a partir de ahí, mediante la ejecución de las transiciones, se llega a enumerar todos los marcados posibles de la red. [24]

Debido a la complejidad de la RdP General Refinada (figura 5), solo se presentará un árbol de alcanzabilidad parcial. En este caso se describen los marcados posibles entre el marcado inicial $\left(M_{0}\right)$ que presenta las marcas de la siguiente manera: $P_{4}(3), P_{6}$ (2), $P_{8}(2), P_{9}(2), P_{11}(2)$. La dinámica representada muestra la evolución del sistema pasando por los estados $P_{13}$ (Listo para volar), $P_{14}$ (Vuelo sin fallos), $P_{15}$ (Vuelo con fallos) hasta llegar al estado $P_{30}$ (Retorno al punto de lanzamiento).

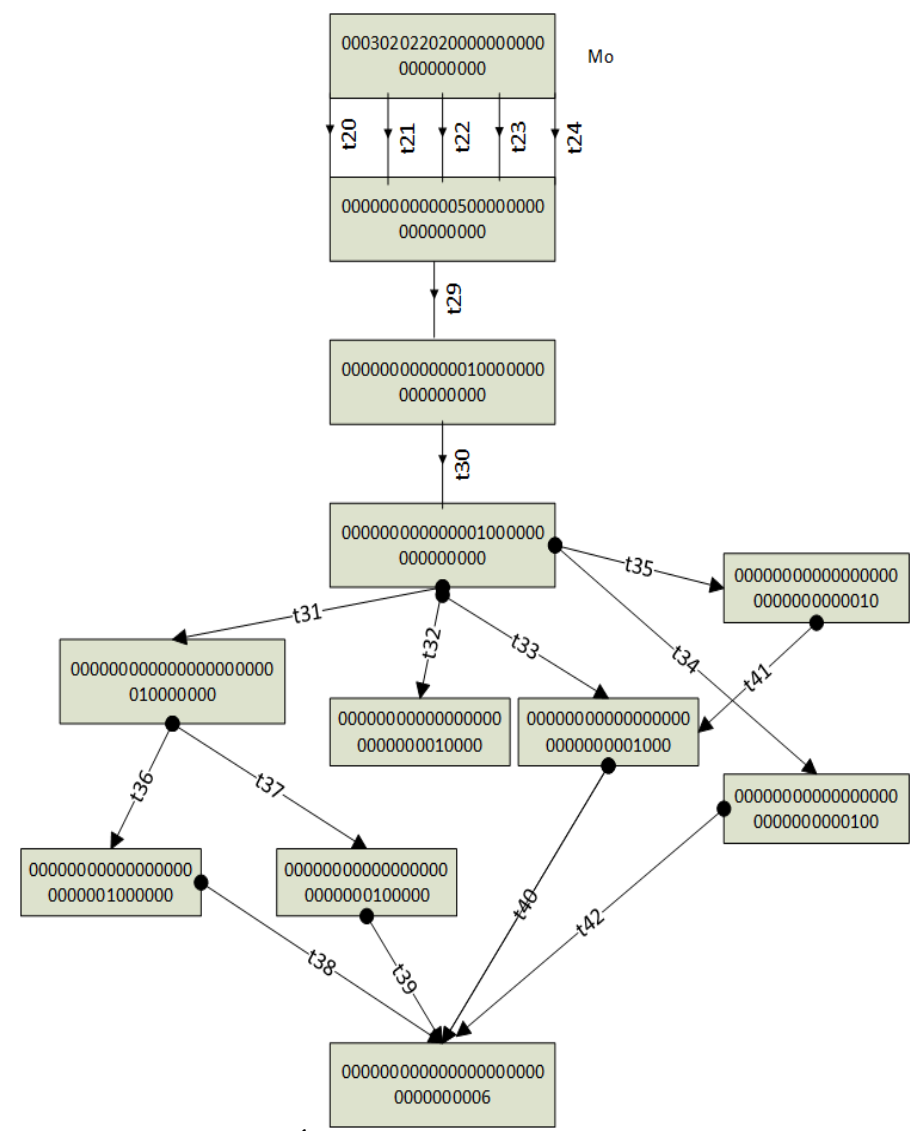

Figura 6: Árbol de alcanzabilidad parcial de la Red de Petri.

Fuente: Autores, (2019).

\section{Ecuación de Estados}

Esta es una ecuación lineal que puede derivarse directamente de la descripción de la red y de su marcado inicial. 
Utilizando este método se puede analizar propiedades como la existencia o no existencia de marcados y/o secuencias de disparos que satisfagan algunas restricciones. [24]

La herramienta PNTool del software MatLab permite obtener Matriz Incidencia $(A)$ de la RdP del VANT. A continuación, se muestra un ejemplo haciendo uso de la ecuación de estados.

El marcado inicial de la RdP está dado por el vector fila

$$
M_{0}=
$$

$[0,0,0,3,0,2,0,2,2,0,2,0,0,0,0,0,0,0,0,0,0,0,0,0,0,0,0,0,0,0]$ que ubica al Quadcopter en un estado de encendido con los chequeos pre-vuelo ya efectuados y comprobados correctamente, el marcado destino $\left(M_{d}\right)$ a alcanzar en el ejemplo será el de estado de Fallos en los subsistemas Motor, Batería y GPS, dado por el vector fila $M_{d}=$ $[0,0,0,2,0,1,0,1,1,0,1,0,4,0,0,1,0,0,0,2,0,1,0,0,0,0,0,0,0,0]$.

En un script de MatLab se definen las traspuestas de estos vectores y se despeja de la ecuación de estado el vector secuencia de disparos $\left(U_{k}\right)$.

$$
U_{k}=
$$

$[0,0,0,0,0,0,0,0,0,0,0,0,0,0,0,0,1,0,2,1,1,1,0,1,0,0,0,0,0,0,0,0,0,0,0$ $, 0,0,0,0,0,0,0,0,0,0,0,0,0,0,0,0,0,0,0,0,0,0,0,0,0,0,0,0,0,0,0,0,0,0,0$ ].

Con estos métodos se pueden verificar las propiedades fundamentales de las RdP, obteniéndose como resultado un modelo general de RdP acotada, viva, libre de interbloqueos y reversible.

\section{CONCLUSIONES}

Se obtuvo un modelo de fallos basado en Redes de Petri que representa el comportamiento normal y de fallo del Quadcopter X4 GARP. El modelo realizado se validó mediante métodos de validación de RdP que permitieron corroborar las propiedades fundamentales del modelado de RdP. Con el modelo propuesto se podrá realizar de forma offline un análisis del comportamiento del VANT a partir de los datos provenientes del controlador de vuelo y detectar los fallos y sus relaciones con otras partes del vehículo con el objetivo de obtener un sistema que permita determinar con mayor facilidad el componente que fallo y sus posibles causas.

\section{REFERENCIAS}

[1] I. Redondo, A. Fernández-Manso, C. Quintano, and L. Calvo, "Diseño e implementación de un VANT (Vehículo Aéreo NoTripulado, Unmanned Aerial Vehicule, UAV) multisensor para estudios post-incendio en entornos forestales," in Congreso Forestal Español, 2018.

[2] K. J. Hayhurst, J. M. Maddalon, P. S. Miner, M. P. DeWalt, and G. F. McCormick, "Unmanned aircraft hazards and their implications for regulation," in 25th Digital Avionics Systems Conference, 2006 IEEE/AIAA, 2006, pp. 1-12.

[3] A. Johry and M. Kapoor, "Unmanned Aerial Vehicle (UAV): Fault Tolerant Design," Int. J. Eng. Technol. Sci. Res., vol. 3, no. 6, pp. 1-7, 2016.

[4] R. Mahony, V. Kumar, and P. Corke, "Multirotor aerial vehicles," IEEE Robot. Autom. Mag., vol. 20, no. 32, 2012.

[5] R. Telford and S. Galloway, "Fault classification and diagnostic system for unmanned aerial vehicle electrical networks based on hidden Markov models," IET Electr. Syst. Transp., vol. 5, no. 3, pp. 103-111, 2015.
[6] A. Mancini, F. Caponetti, A. Monteriu, E. Frontoni, P. Zingaretti, and S. Longhi, "Safe flying for an UAV Helicopter," in Control \& Automation, 2007. MED'07. Mediterranean Conference on, 2007, pp. 1-6.

[7] P. Bauer, R. Venkataraman, B. Vanek, P. J. Seiler, and J. Bokor, "Fault Detection and Basic In-Flight Reconfiguration of a Small UAV Equipped with Elevons," IFAC-Pap., vol. 51, no. 24, pp. 600-607, 2018.

[8] Y. Zhong, Y. Zhang, W. Zhang, J. Zuo, and H. Zhan, "Robust Actuator Fault Detection and Diagnosis for a Quadrotor UAV With External Disturbances," IEEE Access, vol. 6, pp. 48169-48180, 2018.

[9] M. H. Olyaei, H. Jalali, A. Noori, and N. Eghbal, "Fault Detection and Identification on UAV System with CITFA Algorithm Based on Deep Learning," in Electrical Engineering (ICEE), Iranian Conference on, 2018, pp. 988-993.

[10] A. Suarez, G. Heredia, and A. Ollero, "Cooperative Virtual Sensor for Fault Detection and Identification in Multi-UAV Applications," J. Sens., vol. 2018, 2018.

[11] M. Marante-Valdivia and W. Santana-Méndez, "Business Process Management. Conceptual approach,” 2014.

[12] H. A. Florez-Celis, C. A. Ruiz-Zea, G. D. Zapata-Madrigal, and L. A. Martinez-Giraldo, "Maintenance scheduling for a power system operating assets using Petri nets integration with ant colony optimization," in Communications and Computing (COLCOM), 2016 IEEE Colombian Conference on, 2016, pp. 1-6.

[13] R. O. Gonzalez, G. G. Gonzalez, J. Escobar, and R. Y. Barazarte, "Applications of Petri nets in electric power systems," in Central America and Panama Convention (CONCAPAN XXXIV), 2014 IEEE, 2014, pp. 1-6.

[14] Y. Zhang et al., "A fuzzy Petri net based approach for fault diagnosis in power systems considering temporal constraints," Int. J. Electr. Power Energy Syst., vol. 78, pp. 215-224, 2016.

[15] M. P. Cabasino, A. Giua, and C. Seatzu, "Diagnosability of discrete-event systems using labeled Petri nets," IEEE Trans. Autom. Sci. Eng., vol. 11, no. 1, pp. 144-153, 2014.

[16] F. G. Cabral, M. V. Moreira, O. Diene, and J. C. Basilio, “A Petri net diagnoser for discrete event systems modeled by finite state automata," IEEE Trans. Autom. Control, vol. 60, no. 1, pp. 59-71, 2015.

[17] A. Giua and C. Seatzu, "Petri nets for the control of discrete event systems," Softw. Syst. Model., vol. 14, no. 2, pp. 693-701, 2015.

[18] G. Zhu, Z. Li, and N. Wu, "Model-based fault identification of discrete event systems using partially observed Petri nets," Automatica, vol. 96, pp. 201-212, 2018.

[19] A. Morales Varela, J. A. Rojas Ramírez, L. H. Hernández Gómez, Á. Morales González, and M. Y. Jiménez Reyes, "Modelo de un sistema de producción esbelto con redes de Petri para apoyar la toma de decisiones," Ingeniare Rev. Chil. Ing., vol. 23, no. 2, pp. 182-195, 2015.

[20] A. Bertolaso, M. M. Raeissi, A. Farinelli, and R. Muradore, "Using Petri Net Plans for Modeling UAV-UGV Cooperative Landing.," in ECAI, 2016, pp. 1720-1721. 
[21] M. A. Trigos, A. Barrientos, and J. del Cerro, "Diagnóstico de fallas de helicópteros no tripulados basado en redes Petri.," Rev. Investig., vol. 8, no. 2, pp. 91-103, 2016.

[22] M. A. Trigos, A. Barrientos, and J. del Cerro, "Systematic process for building a fault diagnoser based on Petri nets applied to a helicopter," Math. Probl. Eng., vol. 2015, 2015.

[23] C. Cuerno Rejado, L. Garcia Hernandez, A. Sanchez Carmona, A. Carrió Fernández, J. L. Sanchez Lopez, and P. Campoy Cervera, "Evolución histórica de los vehículos aéreos no tripulados hasta la actualidad," Dyna, vol. 91, no. 3, pp. 282-288, 2016.

[24] C. Castellanos, "Consideraciones para el modelado de sistemas mediante Redes de Petri," Cienc. EIng., vol. 27, no. 2, pp. 49-58, 2006. 